\title{
An Appraisal on the Judgements of the Kumamoto Minamata Disease Certification Commission
}

\author{
Masaya MIYAI \\ College of Liberal Arts, Himeji Dokkyo University, Himeji
}

\begin{abstract}
Medical records of 3,870 applicants living near the Shiranui sea coast who had been examined from August 1975 to April 1981 by the Committee on Certification of Minamata Disease were analyzed from the statistical point of view. The author analyzed the pattern of signs and reevaluated the applicants' records.

Three hundred forty applicants (8.8\%) were certified to have Minamata disease by the Committee on Certification of Minamata Disease. By application of the present criteria presented by the Japanese Environmental Agency in 1977 to the records, 947 inhabitants (27.9\%) were judged likely to have Minamata disease.

The author concluded that the certification of the patients of Minamata disease by the Committee was inconsistent with the results of applying the present criteria to the data and that the number of patients certified by the Committee to have Minamata disease was too low.
\end{abstract}

Jpn J Hyg 1997; 51: 711-21.

\section{Analysis of Flushing Response to Alcohol and Drinking Behavior in Japanese Male Students}

\author{
Ikuko SOBUE*1, Hiromi HAYASHI*2, Chizuru MUKAI*2, \\ Tatsuya TAKESHITA*3 and Kanehisa MORIMOTO*3 \\ ${ }^{* 1}$ College of Medical Technology, Kyoto University, Kyoto, ${ }^{* 2}$ Kyoto University Hospital, Kyoto \\ ${ }^{*}$ Department of Hygiene and Preventive Medicine, Osaka University Medical School, Suita
}

\begin{abstract}
The purpose of this study is to clarify the relationship between hereditary predisposition and social-psychological factors in terms of drinking behavior of Japanese male students.

The subjects were 123 male students who belonged to athletic clubs of K university in 1993. Regular drinking was evaluated as "drinking at home or with intimate friends" and party drinking as "drinking at club parties." On the two drinking occasions, the frequency and amount of drinking, and social drinking behavior were evaluated. Problem drinking behavior was investigated by a modified version of Aoyama's questionnaire (1984).
\end{abstract}

Low Km aldehyde dehydrogenase (ALDH2) activity was evaluated by the facial flushing response to alcohol and the ethanol patch test.

Evaluable results of the ethanol test as well as replies of the questionnaire were obtained from 109 students. Twenty-three students positive for both the facial flushing response to alcohol and the ethanol patch test were considered to be flushers, and 42 negative for both to be non-flushers.

The non-flushers consumed a higher amount of alcohol than the flushers did on drinking occasions at home or with intimate friends. The number of problem drinking behavior items in the non-flushers was significantly higher than that in the flushers and increased with the amount and frequency of drinking. The ratio of students who had inappropriate drinking motivations, began to drink by themselves, or continued to offer alcohol to drinkers showing flushing even when they declined it, was significantly higher in the non-flushers than in the flushers. In addition, non-flushers showing such social drinking behavior drank more than those not showing such behavior.

The frequency of positive or inappropriate drinking behavior was significantly lower in the flushers than in the non-flushers. However, the amount and frequency of drinking and problem drinking behavior were similar in the flushers and nonflushers.

These results indicate that social drinking behavior of non-flushers influences not only themselves but also other drinkers, and that drinking of flushers is strongly influenced by social factors.

Jpn J Hyg 1997; 51: 722-33. 


\title{
Effects of Voluntary Exercise on Nonspecific Immunological Mechanisms in Mice
}

\author{
Haruo SUGIURA*1,*2, Hiroyuki NISHIDA*1, Hiroko SUGIURA*2, \\ Ryoichi INABA*2 and Hirotoshi IWATA*2 \\ ${ }^{*}$ Department of Health and Physical Education, Gifu Pharmaceutical University, Gifu \\ *2 Department of Hygiene, Gifu University School of Medicine, Gifu
}

\begin{abstract}
We studied the effects of voluntary exercise on nonspecific immunological mechanisms in mice.
In this study, 7 weekold male ICR mice were divided into two groups: a non-exercise group (control) and a group given voluntary exercise (Vex group). Each mouse of the Vex group was kept in an individual cage equipped with a voluntarily revolving wheel that the mouse had free access to. The duration of voluntary exercise was 3 days per week for 8 weeks.

The following results were obtained:

1) After 8 weeks of voluntary exercise, food consumption, the weight of the anterior tibialis muscle and succinate dehydrogenase activity in the anterior tibialis muscle increased significantly in the Vex group compared to the control group.

2) By means of the carbon clearance method, phagocytosis of the reticuloendothelial system was increased in the Vex group.

3) Glucose consumption capacity and $\mathrm{O}_{2}^{-}$production capacity of peritoneal macrophages $(\mathrm{M} \phi)$ were significantly increased in the Vex group compared to the control group.

4) The acid phosphatase (APH), $\beta$-glucuronidase (GLU) and lactate dehydrogenase (LDH) activities of peritoneal $\mathrm{M} \phi$ increased significantly in the Vex group.

5) Concanavalin A (Con A)-induced cell proliferatoin in the spleen was high in the Vex group.

Based on the above findings, it may be surmised that voluntary exercise enhances nonspecific immunological mechanisms and thereby improves the host defense mechanisms in mice.
\end{abstract}

Jpn J Hyg 1997; 51: 734-41.

\section{Investigation of Bone Mineral Distribution in Japanese Using Dual-energy X-ray Absorptiometry}

\author{
Chang-nian WEI*1, Hiroyuki YONEMITSU*1, Hidetaro SHIBAYAMA*2 \\ and Atsushi UEDA*3 \\ ${ }^{* 1}$ Kumamoto Kinoh Hospital, Kumamoto \\ *2 National Institute of Fitness and Sports in Kanoya, Kanoya \\ ${ }^{* 3}$ Department of Hygiene, Kumamoto University School of Medicine, Kumamoto
}

\begin{abstract}
To investigate bone mineral distribution in humans, the authors conducted a cross-sectional survey of, and performed bone-density measurements on, 1,310 healthy Japanese ranging in age 5 to 85 years. Eight hundred fifty-eight of the subjects were female, and 452 were male. Arm, leg, and spine bone mineral content (BMC) and bone mineral density (BMD) were assessed by dual-energy X-ray absorptiometry (DXA), and the subjects were divided into 5-year age groups. BMD showed increases with skeletal growth until reaching a peak at 15 to 19 years in females, and 25 to 29 for males. For both sexes the fastest growth to maturity in terms of bone mass values was in the late $20 \mathrm{~s}$. Females, though, had higher arm, leg, and spine remodeling rates than males. In premenopausal women no changes in arm, leg or spine BMC and BMD were observed. Postmenopausal women showed an overall reduction in bone mass, most noticeably in the spine. After menopause, women had about 10 years of accelerated loss (1.46\%/year). Vertebral BMD values were similar for men and women $\left(1.10 \pm 0.20 \mathrm{~g} / \mathrm{cm}^{2}\right.$ for males vs. $1.09 \pm 0.14 \mathrm{~g} / \mathrm{cm}^{2}$ for females, $\left.\mathrm{p}>0.05\right)$. BMC values were significantly higher in males, and males at all times had a higher arm and leg BMD. There were no significant value differences in either sex for left and right leg $\mathrm{BMC}$ and $\mathrm{BMD}$; however, from the age of 15 , right arm values were significantly higher likely due to right handedness. For both sexes the order of BMC and BMD was leg, spine, and arm.
\end{abstract}

Jpn J Hyg 1997; 51: 742-8. 


\title{
Reduction of Alcohol Consumption and its Effect on $\mathrm{Na}^{+}-\mathrm{K}^{+}$-ATPase Activity of Red Blood Cell Membrane
}

\author{
Yutaka KUROSAWA*1, Yuchi NARUSE*1, Sadanobu KAGAMIMORI*1 \\ Kazuo MIKAWA ${ }^{* 2}$ and Hirotsugu UESHIMA*3 \\ ${ }^{* 1}$ Department of Community Medicine, Toyama Medical and Pharmaceutical University, Toyama \\ ${ }^{* 2}$ Department of Medical Affair, Daido Mutual Life Insurance Company, Osaka \\ ${ }^{* 3}$ Department of Health Science, Shiga University of Medical Science, Shiga
}

\begin{abstract}
Fifty-four untreated, mildly hypertensive men who were civil servants and were aged 30-59 years reduced their alcohol consumption as much as possible for three weeks. Then blood pressure and serum gamma GTP decreased significantly, but $\mathrm{Na}^{+}-\mathrm{K}^{+}$-ATPase activity of the red blood cell membrane did not change significantly. The enzyme activity in twenty subjects ( $45.5 \%$ of total subjects) increased and that in twenty-four subjects (54.5\%) decreased or was unchanged. No significant relationship between the ratio of the change of $\mathrm{Na}^{+}-\mathrm{K}^{+}$-ATPase activity and that of blood pressure or serum gamma GTP was observed.
\end{abstract}

Jpn J Hyg 1997; 51: 749-54.

\section{Bone Mineral Density by Ultrasonic Measurement in Pre- and Postmenopausal Women Relationship with Sex Hormones and Nutritional States}

\author{
Naoko SHONO*1, Kenji KUGINO*2, Setsuko YOSHIDA*2, \\ Mioko NAKAYAMA*2, Hirofumi UENO $^{* 3}$ and Masahiro NISHIZUMI ${ }^{* 1}$ \\ *1 Department of Community Health Science, Saga Medical School, Saga \\ ${ }^{* 2}$ Department of Nutrition, Yamaguchi Prefectural University, Yamaguchi \\ ${ }^{* 3}$ Saga Research Institute, Otsuka Pharmaceutical Co., Ltd., Saga
}

\begin{abstract}
In this cross-sectional study we evaluated the relationships of the sex hormones, estradiol $\left(\mathrm{E}_{2}\right)$, free testosterone (free T), dehydroepiandrosterone-sulfate (DHEA-S), sex hormone-binding globulin (SHBG) and nutritional status to bone density assessed by ultrasonic measurements. The subjects were 16 premenopausal (38-50 years) and 28 postmenopausal (47-86 years) women living in a rural area in Yamaguchi Prefecture in Japan. They were healthy and did not smoke or drink. Speed of sound (SOS), broadband ultrasound attenuation (BUA) and the stiffness index (SI) were used as the indices to evaluate ultrasonic bone density (UBD) for the calcaneus.

In premenopausal women, the concentration of free $T$ was negatively associated with BUA. The DHEA-S concentration had a significant negative correlation with BUA after adjustments for age and body mass index (BMI). Daily intakes of fat, animal fat, animal protein, calcium $(\mathrm{Ca})$ and vitamin $\mathrm{B}_{2}\left(\mathrm{Vit}_{\mathrm{i}} \mathrm{B}_{2}\right)$ were positively associated with UBD. In postmenopausal women, although DHEA-S positively correlated with SOS, BUA and SI, the significance disappeared after adjustments for age and BMI. The protein intake was positively associated with BUA.

Multiple regression analysis for SOS, BUA and SI as dependent variables was performed. Independent variables were age, $B M I, E_{2}$, free T, DHEA-S, SHBG and daily intakes of animal fat, animal protein, $V_{i t} B_{2}$ and $C a$. In premenopausal women, the animal fat intake contributed significantly and positively to the variations of SOS and SI. The intake of Vit $\mathrm{B}_{2}$ had a significant positive correlation, and the level of DHEA-S had a significant negative correlation with BUA. In postmenopausal women, age and BMI contributed significantly to the variations of SOS, BUA and SI, while the nutritional variables and the sex hormones were not found to be significant.

In conclusion, different factors predictiong UBD were suggested for pre-and postmenopausal women. In premenopausal women, daily intakes of animal fat and $V_{i t} B_{2}$ might be effective to maintain higher bone density. Further investigations are still needed to clarify the relationship between DHEA-S and UBD.
\end{abstract}




\title{
Aluminum Contents in Dried and Cooked Sea Vegetables
}

\author{
Fumiko MATSUSHIMA*1, Shunsuke MESHITSUKA*2 and Takayuki NOSE*3 \\ *1 Tottori Women's College, Kurayoshi \\ *2 Department of Health Systems and Informatics, Faculty of Medicine, Tottori University, Yonago \\ ${ }^{*}$ Department of Public Health, Faculty of Medicine, Tottori University, Yonago
}

\begin{abstract}
Regarding aluminum contents in sea vegetables, dried hijiki (an edible brown algae), dried wakame (seaweed) and dried konbu (kelp) contained $1.08 \mathrm{mg} / \mathrm{g}, 17.7$ to $149 \mu \mathrm{g} / \mathrm{g}$, and 4.23 to $24.5 \mu \mathrm{g} / \mathrm{g}$, of aluminum, respectively. With hijiki, especially, having a high aluminum content. Hijiki reconstituted by water immersion contained 450 to $678 \mu \mathrm{g} / \mathrm{g}$, of aluminum. Thus, aluminum in dried hijiki was decreased by 40 to $66 \%$ by water immersion. The immersion water of blanched and salted wakame and fruit-bearing leaf wakame contained 4.67 to $6.92 \mu \mathrm{g}$ and $1.00 \mu \mathrm{g}$ of aluminum, respectively. The migration ratio of aluminum was $8-12 \%$ for blanched and salted wakame and was $1 \%$ for fruit-bearing leaf wakame. The immersion water of Hidaka konbu contained 16.1 to $21.4 \mu \mathrm{g}$ of aluminum, while that of Rishiri konbu contained 4.79 to $7.36 \mu \mathrm{g}$ of aluminum. Thus, 22 to $57 \%$ of aluminum migrated into the konbu immersion water. Aluminum scarcely migrated into the supernatant of the immersion water. Compared with the cold water immersion method, a high aluminum migration ratio was achieved in the heat extraction method. It was estimated that the aluminum intake from reconstituted hijiki (dry weight: $5 \mathrm{~g}$ ) was about 2.2 to $3.4 \mathrm{mg}$, which amounted to 50 to $75 \%$ of the daily aluminum intake of a typical Japanese diet.
\end{abstract}

Jpn J Hyg 1997; 51: 763-9.

\section{Interrelations of the Calcium Concentration in Breast Milk with Maternal Intake of Cow's Milk and Milk Products, Bone Resorption and Bone Mineral Density during Lactation}

\author{
Kyoko YONEYAMA*1, Junko IKEDA*2 and Hisanori NAGATA*3 \\ ${ }^{* 1}$ Nara University of Education, Nara ${ }^{* 2}$ Kyoto Bunkyo College, Uji \\ ${ }^{*}$ Mukogawa Women's University, Nishinomiya
}

\begin{abstract}
The interrelations of the calcium (Ca) concentration in breast milk with maternal intake of cow's milk and milk products, 24-hour urinary hydroxyproline and $\mathrm{Ca}$ concentrations adjusted by creatinine $(\mathrm{H} . \mathrm{P} / \mathrm{Cre}, \mathrm{Ca} / \mathrm{Cre})$ and bone mineral density measured by ultrasonic bone densitometry were examined to study the mechanism regulating the calcium concentration in breast milk. Subjects were 105 lactating women, aged 21 to 42 years, at 21-590 days postpartum.

1) The geometric means (logarithmically transformed calculated $M \pm S D$ ) of H.P/Cre were 40.7 (31-61) and 36.3 (28-47) $\mathrm{mg} / \mathrm{g}$ in women lactating less than 150 days and 150 days or more, respectively. These values indicated increased bone resorption.
\end{abstract}

2) Intake of cow's milk and milk products significantly negatively correlated with urinary H.P/Cre. For women consuming less than $100 \mathrm{ml}$ per day of cow's milk, a significant positive correlation was found between the urinary $\mathrm{H} . \mathrm{P} / \mathrm{Cre}$ and $\mathrm{Ca}$ concentration in breast milk. These results suggest that the extent of bone resorption depends on maternal $\mathrm{Ca}$ intake and the $\mathrm{Ca}$ transfer from maternal bone to breast milk in women with a low Ca intake.

3) For women with a cow's milk intake of less than $100 \mathrm{ml}$ per day, a significant positive correlation was found between the Ca concentration in breast milk and bone mineral density, indicating that women with bone mineral loss are not able to compensate for the low Ca level in breast milk.

4) Urinary $\mathrm{Ca} / \mathrm{Cre}$ significantly negatively affected the $\mathrm{Ca}$ concentration in breast milk. This result indicates that some amount of $\mathrm{Ca}$ in breast milk is due to renal conservation.

Jpn J Hyg 1997; 51: 770-9. 